\title{
A Novel Capacitive Detection Scheme With Inherent Self-Calibration
}

\author{
Alexander A. Trusov, Student Member, IEEE, Student Member, ASME, and \\ Andrei M. Shkel, Associate Member, IEEE, Associate Member, ASME
}

\begin{abstract}
This paper reports a novel capacitive detection method, called the sideband-ratio (SBR) detection, which is robust to variations of such critical parameters as the nominal capacitance, frequency, and amplitude of the probing voltage and gain of the transimpedance amplifier. The approach constructively utilizes the inherent nonlinearity of parallel-plate sense capacitors in order to measure the amplitude of sinusoidal motion. In the case of parallel-plate detection signal, multiple harmonics exist and each carry information about the amplitude of mechanical motion. The SBR method robustly extracts the amplitude of motion from the ratio of multiple sidebands. This paper presents theoretical background and analysis of this nonlinear measurement method. A real-time measurement algorithm is implemented in software and simulated. Feasibility of the developed approach is demonstrated experimentally.

[2006-0292]
\end{abstract}

Index Terms-Automatic calibration, capacitive detection, electromechanical amplitude modulation (EAM), gyroscopes, modulation, parallel plates, resonators, self-calibration, sidebands.

\section{INTRODUCTION}

M ICROMACHINED gyroscopes [1], filters [2], [3], mixlers [4], and various other dynamic microelectromechanical-system (MEMS) devices [5] utilize resonant structures in their operation. In all these devices, the vibratory motion in one or more modes needs to be actuated, detected, and, in some cases, controlled. This paper focuses on capacitive detection of oscillations utilizing signal processing of the motion-induced current. Conventional capacitive-detection schemes produce a signal proportional to such system parameters as nominal sense capacitance, carrier voltage amplitude and frequency, and gain of the current amplifier. These dependencies constitute a need to periodically calibrate individual MEMS devices to address mechanical-element and electronicsfabrication imperfections and fluctuation of the parameters due to changing environment and aging. A detection technique independent of these system parameters can be of a great advantage.

Capacitive detection of motion is usually based on measuring the current induced by the relative motion between a mobile

Manuscript received December 31, 2006; revised July 23, 2007. This work was supported in part by the National Science Foundation under Grant CMS-0409923, in part by BEI Technologies under Contract BEI-36974, and in part by UC Discovery Program ELE04-10202. Subject Editor S. Merlo.

The authors are with the Mechanical and Aerospace Engineering Department, MicroSystems Laboratory EG2110, University of California, Irvine, CA 92697 USA (e-mail: atrusov@uci.edu; ashkel@uci.edu).

Digital Object Identifier 10.1109/JMEMS.2007.906077 and an anchored electrode forming a variable sense capacitor. This capacitor is biased by a certain known dc or ac voltage [6]-[8]. The motion-induced change in capacitance results in the flow of current, which is converted to a voltage using a transimpedance amplifier. This output voltage is detected and processed in order to measure the motion of the vibrating structure. Electromechanical amplitude modulation (EAM) is a widely used capacitive detection approach. It is based on the modulation of the motional signal by an ac-probing voltage (carrier) and allows for frequency-domain separation between the informational signals and feed-through of the driving voltages [6], [9]. The conventional linear-EAM approach can be used with either lateral-comb sense or small-displacement parallel-plate capacitors. In the linear case, only one pair of amplitude-modulated sidebands exists and external calibration of the pick-up signal is needed to identify the voltage-todisplacement scaling factors of the detection channels.

The topic of signal nonlinearity in parallel-plate-detection schemes was introduced in [10]. In this paper, we develop a novel capacitive detection and self-calibration method that takes advantage of the parallel-plate nonlinearity to eliminate the effect of the nominal capacitance, probing voltage, and the transimpedance gain on the measurement. First, we describe the electromechanical model of a resonator with parallel-plate capacitive detection in Section II. The parallel-plate EAM pickup signal is studied in Section III. Based on the nonlinear features of the pick-up signal, the sideband-ratio (SBR) detection approach is formulated in Section IV and verified experimentally in Section V. The complete real-time measurement algorithm based on the SBR is presented in Section VI along with simulation results. Section VII reports the adaptation of the method for differential EAM and pure dc detection, approaches to sidebands demodulation, and noise-performance analysis. Section VIII discusses applications of the SBR method and concludes this paper.

\section{Electromechanical Model}

Fig. 1 shows a general schematic of a capacitive microresonator utilizing parallel-plate SBR-detection scheme. The electromechanical diagram includes the mechanical resonator, the lateral-comb drive and parallel-plate sense electrodes, and a signal-processing block for detection of motion. The suspended mass of the resonator is constrained to move only along the horizontal $x$-axis. The variable sense capacitance is defined as $C_{\mathrm{s}}(x)$, and the drive capacitance as $C_{\mathrm{d}}(x)$, where $x$ is the displacement. Typically, in MEMS devices, drive and sense 


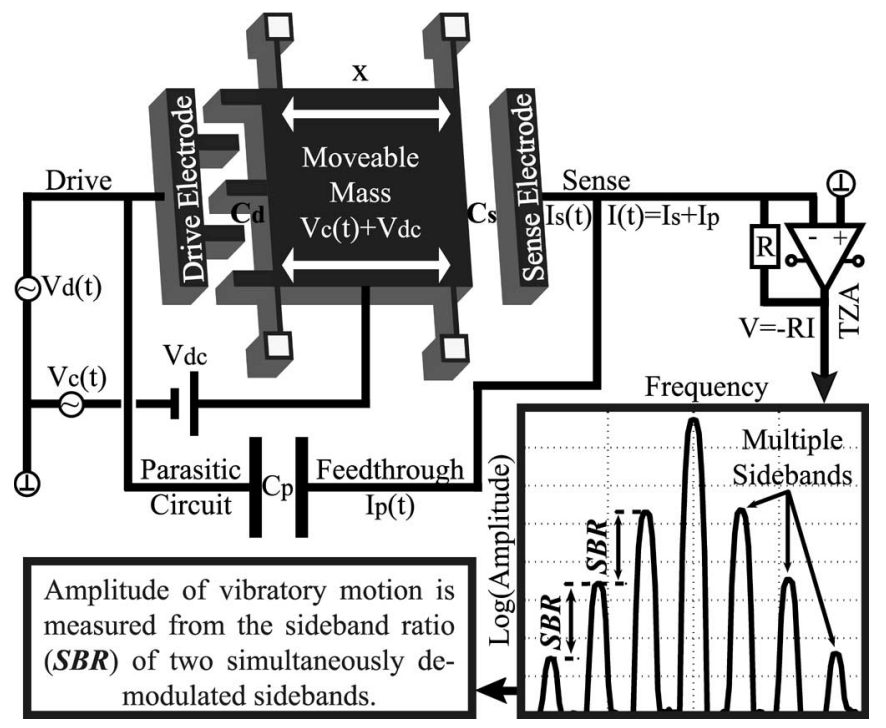

Fig. 1. Schematics of a capacitive MEMS resonator with parallel-plate detection of motion.

terminals are not completely isolated but are electrically coupled by stray parasitic capacitances and resistances [8]. In this paper, we assume, without loss of generality, that the parasitic circuit consists of a single lumped capacitor $C_{\mathrm{p}}$. An ac driving voltage $V_{\mathrm{d}}(t)=v_{\mathrm{d}} \cos \left(\omega_{\mathrm{d}} t\right)$ is applied to the drive electrode (voltage values are referenced with respect to a common ground). The sense capacitor $C_{\mathrm{s}}(x)$ is formed between the mobile mass and the fixed sense electrode. The sense electrode is connected to the inverting input of an operational amplifier, which is configured as a transimpedance amplifier [11].

The vibratory motion at the drive frequency $\omega_{\mathrm{d}}$ is excited by a combination of $V_{\mathrm{dc}}$ and $V_{\mathrm{d}}$ driving voltages across the drive capacitor. Without discussing further details of the actuation scheme, the resonator's displacement $x(t)$ can be expressed as

$$
x(t)=\|x\| \sin \left(\omega_{\mathrm{d}} t+\phi\right)
$$

where $\phi=\phi\left(\omega_{\mathrm{d}}\right)$ represents a phase lag of the transfer function of the resonator. The capacitive detection results presented in this paper are, in fact, valid for any microstructure with sinusoidal motion that is independent of the actuation mechanism.

Due to the sinusoidal motion, the sense capacitance $C_{\mathrm{s}}(x)$ changes, causing a flow of motional current $I_{\mathrm{s}}(t)=$ $d\left(C_{\mathrm{s}} V_{\mathrm{s}}\right) / d t$, where $V_{\mathrm{s}}$ is the probing voltage across the sense capacitor. The total pick-up current $I(t)=I_{\mathrm{s}}(t)+I_{\mathrm{p}}(t)$ consists of both the motional and the parasitic currents and is converted to the final output voltage $V(t)$ with transimpedance gain $-R$. Parasitic current is induced by the drive voltage $V_{\mathrm{d}}$ and, therefore, has the same frequency $\omega_{\mathrm{d}}$. In this paper, we assume that the total sensing voltage $V_{\mathrm{s}}(t)=V_{\mathrm{dc}}+V_{\mathrm{c}}(t)$ is composed of a dc component $V_{\mathrm{dc}}$ and an ac component $V_{\mathrm{c}}=$ $v_{\mathrm{c}} \sin \left(\omega_{\mathrm{c}} t\right)$, which is called the carrier. Particular cases when either the dc or ac component of the sense voltage is zero are discussed later in this paper. Use of an ac carrier voltage results in an amplitude modulation of the motional signal, which is known as EAM.
The total pick-up voltage on the output of the current amplifier is given by

$$
V(t)=-R \frac{d}{d t}\left[V_{\mathrm{d}}(t) C_{\mathrm{p}}+\left(V_{\mathrm{c}}(t)+V_{\mathrm{dc}}\right) C_{\mathrm{s}}(t)\right] .
$$

In Section III, we expand the pick-up signal for the case of parallel-plate sense capacitor without using a smalldisplacement assumption.

\section{Motion Detection With Parallel Plates}

This section studies the nonlinear properties of parallel-plate EAM pick-up signal. Consider a variable sense capacitor $C_{\mathrm{s}}(x)$ formed by a pair of mobile and anchored parallel-plate structures. Let us denote permittivity of media by $\varepsilon$, the initial gap between plates at rest by $g$, the overlap length of an individual parallel-plate pair by $L$, and the height of the plates (i.e., the structural layer thickness) by $y$. The total overlap area in the sense capacitor is given by $A=N L y$, where $N$ is a number of parallel-plate pairs in the capacitor. The total variable sense capacitance for the sinusoidal mode of motion in (1) is

$$
C_{\mathrm{s}}(x(t))=\frac{\varepsilon A}{g-x(t)}=\frac{\varepsilon A}{g} \frac{1}{1-\frac{\|x\|}{g} \sin \left(\omega_{\mathrm{d}} t\right)}
$$

where the phase of motion $\phi$ is omitted without any loss of generality.

We introduce the nominal sense capacitance $C_{\mathrm{sn}}=\varepsilon A / g$ and dimensionless amplitude of motion $x_{0}=\|x\| / g<1$ normalized with respect to the initial gap between the parallel plates. From (3), the sense capacitance is

$$
C_{\mathrm{s}}(t)=C_{\mathrm{sn}} \frac{1}{1-x_{0} \sin \left(\omega_{\mathrm{d}} t\right)} .
$$

As shown in Appendix I, the Fourier-series representation of the parallel-plate capacitance $C_{\mathrm{s}}(t)$ for a given amplitude of motion $x_{0}$ is

$$
\begin{aligned}
C_{\mathrm{s}}(t)=C_{\mathrm{sn}} \sum_{k=0}^{\infty} p_{2 k}\left(x_{0}\right) \cos \left(2 k \omega_{\mathrm{d}} t\right) \\
\quad+C_{\mathrm{sn}} \sum_{k=0}^{\infty} p_{2 k+1}\left(x_{0}\right) \sin \left((2 k+1) \omega_{\mathrm{d}} t\right)
\end{aligned}
$$

where functions $p_{k}\left(x_{0}\right)$ define the amplitudes of the multiple harmonics of the motional frequency in the capacitance $C_{\mathrm{s}}(t)$. The amplitudes form a geometric progression and, in closedform, are given by

$$
\begin{aligned}
\left\|p_{k}\left(x_{0}\right)\right\| & =\frac{2}{\sqrt{1-x_{0}^{2}}}\left(\frac{x_{0}}{\left(1+\sqrt{1-x_{0}^{2}}\right)}\right)^{k} \\
& =2 p_{0}\left(x_{0}\right)\left(\frac{x_{0}}{\left(1+\sqrt{1-x_{0}^{2}}\right)}\right)^{k} .
\end{aligned}
$$




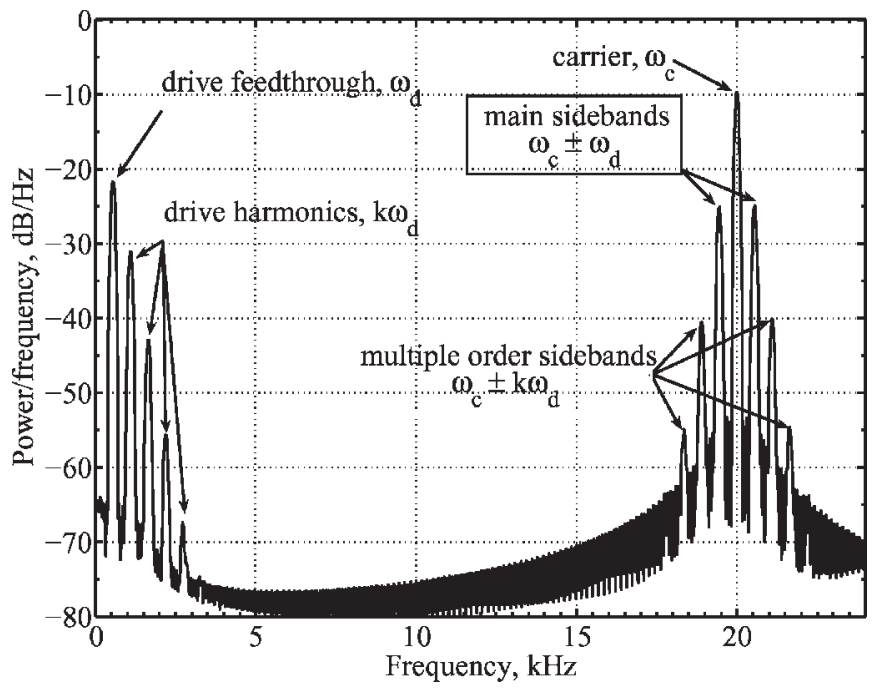

Fig. 2. PSD of a parallel-plate EAM pick-up signal, computer simulation.

Equation (5) shows that when parallel plates are used to sense a sinusoidal motion, the time-varying sense capacitance contains an infinite number of drive-frequency harmonics. In order to calculate the total output signal, we consider the modulation of each capacitance harmonic individually. We combine the sense capacitance in (5) with (2) to obtain the total output current

$$
\begin{aligned}
I(t)= & -C_{\mathrm{p}} v_{\mathrm{d}} \omega_{\mathrm{d}} \sin \left(\omega_{\mathrm{d}} t\right) \\
+ & C_{\mathrm{sn}} v_{\mathrm{c}} \omega_{\mathrm{c}} p_{0}\left(x_{0}\right) \cos \left(\omega_{\mathrm{c}} t\right)+C_{\mathrm{sn}} V_{\mathrm{dc}} \omega_{\mathrm{d}} \\
& \times\left\{\sum_{k=0}^{\infty}(2 k+1) p_{2 k+1}\left(x_{0}\right) \cos \left((2 k+1) \omega_{\mathrm{d}} t\right)\right. \\
& \left.\quad-\sum_{k=1}^{\infty} 2 k p_{2 k}\left(x_{0}\right) \sin \left(2 k \omega_{\mathrm{d}} t\right)\right\} \\
+ & \frac{1}{2} C_{\mathrm{sn}} v_{\mathrm{c}} \sum_{k=0}^{\infty} p_{2 k+1}\left(x_{0}\right) \\
& \times\left[\omega_{(2 k+1)} \sin \left(\omega_{(2 k+1)} t\right)-\omega_{-(2 k+1)} \sin \left(\omega_{-(2 k+1)} t\right)\right] \\
+ & \frac{1}{2} C_{\mathrm{sn}} v_{\mathrm{c}} \sum_{k=1}^{\infty} p_{2 k}\left(x_{0}\right) \\
& \left.\left.\times\left[\omega_{(2 k)} \cos \left(\omega_{(2 k)}\right) t\right)+\omega_{(-2 k)} \cos \left(\omega_{(-2 k)}\right) t\right)\right]
\end{aligned}
$$

where $\omega_{k}=\omega_{c}+k \omega_{\mathrm{d}}$ is the frequency of the $k$ th-order sideband (left or right, depending on the sign of the index $k$ ). This equation gives the Fourier series of the total pick-up current for the case of parallel-plate capacitive detection of sinusoidal motion. Fig. 2 shows a power-spectral-density (PSD) plot of a typical parallel-plate EAM pick-up signal and illustrates its important features. The signal contains an infinite number of harmonics corresponding to the drive frequency and multiple informational sidebands.
Let $V_{\omega}$ denote the Fourier component of frequency $\omega$ in the total output voltage $V=-R I(t)$. According to (7), the amplitudes of the multiple sidebands in the total output voltage are given by

$$
\left\|V_{\omega_{ \pm k}}\right\|=\frac{1}{2} R C_{\mathrm{sn}} v_{\mathrm{c}}\left\|\left(\omega_{\mathrm{c}} \pm k \omega_{\mathrm{d}}\right) p_{k}\left(x_{0}\right)\right\|
$$

In practice, a high-frequency carrier is usually used [6], [9] so that $\omega_{\mathrm{c}} \gg\|k\| \omega_{\mathrm{d}}$ for several first-order harmonics, $k=$ $\pm 1, \pm 2, \pm 3, \ldots, \pm K$. For these low-order sidebands

$$
\begin{aligned}
\left\|V_{\omega_{ \pm k}}\right\| & =\frac{1}{2} R C_{\mathrm{sn}} v_{\mathrm{c}}\left(\omega_{\mathrm{c}} \pm k \omega_{\mathrm{d}}\right)\left\|p_{k}\left(x_{0}\right)\right\| \\
& \approx \frac{1}{2}\left(R C_{\mathrm{sn}} v_{\mathrm{c}} \omega_{\mathrm{c}}\right)\left\|p_{k}\left(x_{0}\right)\right\| .
\end{aligned}
$$

The amplitudes of the multiple sidebands are proportional to the functions $p_{k}\left(x_{0}\right)$, which we thus call the normalized sidebands amplitudes. For a given motional amplitude $x_{0}$, the normalized amplitudes of the multiple sidebands form a geometric progression with the ratio

$$
r\left(x_{0}\right)=\left\|p_{k+1}\left(x_{0}\right)\right\| /\left\|p_{k}\left(x_{0}\right)\right\|=\frac{x_{0}}{1+\sqrt{1-x_{0}^{2}}} .
$$

In Section IV, we discuss how simultaneous demodulation of the multiple sidebands can be used to produce a self-calibrated measurement of the motional amplitude $x_{0}$. Section $\mathrm{V}$ provides experimental confirmation of (10).

\section{Demodulation of Parallel-Plate EAM Signal}

In this section, we discuss how the ratio of parallel-plate EAM sidebands can be used to robustly detect the vibratory motion. First, we review a conventional linear approach to the EAM detection as follows [6], [9].

\section{A. Conventional Linear Approach}

In the conventional linear approach (i.e., for lateral-comb sense capacitor or parallel-plate sense capacitor with smallamplitude assumption), only the main, order $k= \pm 1$, sidebands are considered. In addition, the amplitudes of these main sidebands are assumed to be linear with respect to the amplitude of motion, $\left\|V_{\omega_{\mathrm{c}} \pm \omega_{\mathrm{d}}}\right\| \propto x_{0}$. In the conventional approach, the extraction of the motional amplitude from the EAM pick-up voltage consists of amplitude demodulation and scaling steps.

1) Mixing $V(t)$ with phase-shifted carrier signal [i.e., multiplication $\left.V(t) \otimes \sin \left(\omega_{\mathrm{c}} t\right)\right]$ to map the sidebands from $\omega_{\mathrm{c}} \pm \omega_{\mathrm{d}}$ to $\omega_{\mathrm{d}}$ frequency.

2) Low-pass filtering to attenuate signals at frequencies higher than $\omega_{\mathrm{d}}$. 
3) Mixing the resulting signal with $\sin \left(\omega_{\mathrm{d}} t+\alpha\right)$ to map the signal from $\omega_{\mathrm{d}}$ to dc. Phase $\alpha$ needs to be controlled to match the phase of motion; alternatively, a dual-phase I/Q demodulation can be used.

4) The obtained dc signal is scaled by $\left(R C_{\mathrm{sn}} v_{\mathrm{c}} \omega_{\mathrm{c}}\right)^{-1}$ to calculate the amplitude of mechanical motion.

Consider a resonant MEMS device in which the amplitude of motion in a certain vibrational mode needs to be accurately detected. Due to the scaling step 4), the conventional approach produces the measurement based on assumed values of such system parameters as transimpedance gain, nominal capacitance, and carrier voltage amplitude and frequency. Accordingly, each electromechanical device needs to be calibrated to identify these parameters. Moreover, during the operation, some of the parameters are prone to drifts, causing the loss of calibration. Approaches based on only the first-order sidebands do not provide a robust solution to this problem. Additionally, in the case of a parallel-plate sense capacitor, the amplitudes of the informational sidebands are not linear with respect to the amplitude of motion. This nonlinearity introduces a significant systematic error if large amplitude of motion is being measured.

\section{B. SBR-Detection Approach}

In the case of parallel-plate sense capacitors, it is possible to detect arbitrary amplitude of motion without explicitly using the values of $R, C_{\mathrm{sn}}, \omega_{\mathrm{c}}$, and $v_{\mathrm{c}}$. The approach is based on simultaneous processing of multiple sidebands and produces the measurement of $x_{0}$ by using the ratio $r$ of magnitudes of two different order sidebands.

According to (8) and (10), the ratio of amplitudes of any two successive sidebands depends only on the amplitude of motion $x_{0}$, while all other parameters cancel out

$$
\begin{aligned}
\frac{\left\|V_{\omega_{-(k+1)}}\right\|+\left\|V_{\omega_{(k+1)}}\right\|}{\left\|V_{\omega_{-k}}\right\|+\left\|V_{\omega_{k}}\right\|} & =\frac{\left\|p_{k+1}\left(x_{0}\right)\right\|}{\left\|p_{k}\left(x_{0}\right)\right\|}=r\left(x_{0}\right) \\
& =\frac{x_{0}}{\left(1+\sqrt{1-x_{0}^{2}}\right)} .
\end{aligned}
$$

From (9), the following approximation is valid for the ratio of two low-order single-side sidebands:

$$
r\left(x_{0}\right) \approx \frac{\left\|V_{\omega_{\mathrm{c}} \pm(k+1) \omega_{\mathrm{d}}}\right\|}{\left\|V_{\omega_{\mathrm{c}} \pm k \omega_{\mathrm{d}}}\right\|} .
$$

There is a direct one-to-one relationship between the amplitude of motion and the amplitude ratio of any two successive parallel-plate EAM sidebands. Solving (10) for the amplitude of motion yields

$$
x_{0}=\frac{2 r}{r^{2}+1} .
$$

This relationship can be linearized in order to provide a very simple method of $x_{0}$ estimation

$$
x_{0} \approx \widetilde{x_{0}}=2 r
$$

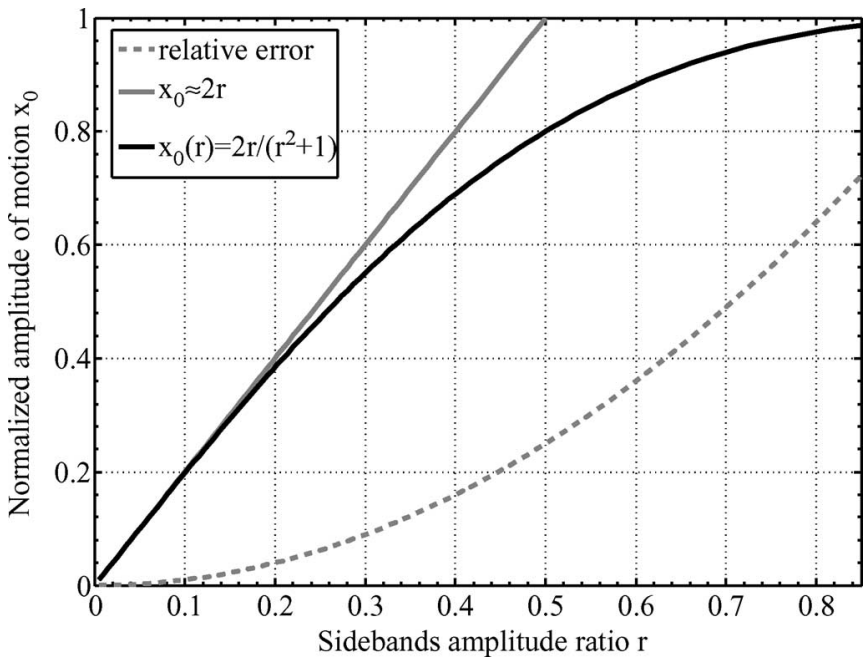

Fig. 3. Detection of the amplitude of motion based on the measured ratio of two sidebands.

with the relative error of the linearization given by

$$
e_{\widetilde{x_{0}}}=\frac{x_{0}-\widetilde{x_{0}}}{x_{0}}=r^{2}
$$

According to this equation, the relative error of linearization $\widetilde{x_{0}}$ is quadratically small for small amplitudes of motion.

Fig. 3 illustrates (13)-(15). The dimensionless amplitude of motion $x_{0}$ can be accurately calculated from the ratio of parallel-plate sidebands using (13) or estimated by (14). The ratio of the sidebands depends only on the amplitude of the motion, while all other common parameters cancel out. A realtime detection algorithm with inherent self-calibration based on the SBR is proposed according to the following procedure:

1) Detect amplitude of the first-order sidebands at frequen$\operatorname{cies} \omega_{\mathrm{c}} \pm \omega_{\mathrm{d}}$.

2) Simultaneously, detect amplitude of the second-order sidebands at frequencies $\omega_{\mathrm{c}} \pm 2 \omega_{\mathrm{d}}$.

3) Calculate the ratio of amplitudes $r=\left(\left\|V_{\omega_{-2}}\right\|+\right.$ $\left.\left\|V_{\omega_{2}}\right\|\right) /\left(\left\|V_{\omega_{-1}}\right\|+\left\|V_{\omega_{1}}\right\|\right) \quad$ or estimate using a single-side pair of sidebands $r \approx\left(\left\|V_{\omega_{-2}}\right\| /\left\|V_{\omega_{-1}}\right\|\right) \approx$ $\left(\left\|V_{\omega_{2}}\right\| /\left\|V_{\omega_{1}}\right\|\right)$.

4) Extract the normalized amplitude of motion $x_{0}=$ $2 r /\left(r^{2}+1\right)$.

Demodulation of the first- and second-order sidebands can be done similarly to the conventional case (see Section VII-C). A complete real-time implementation of the SBR signalprocessing algorithm is presented in Section VI along with simulation results.

\section{ExPerimental Demonstration}

In order to verify the relationship between the amplitude of motion and the ratio of sidebands in (13), test structures were designed, fabricated, and characterized. The devices were capacitive MEMS resonators with lateral-comb drive capacitors and lateral-comb and parallel-plate sense capacitors. The fabrication was done using an in-house wafer-level silicon-oninsulator (SOI) process. SOI wafers with a highly conductive 


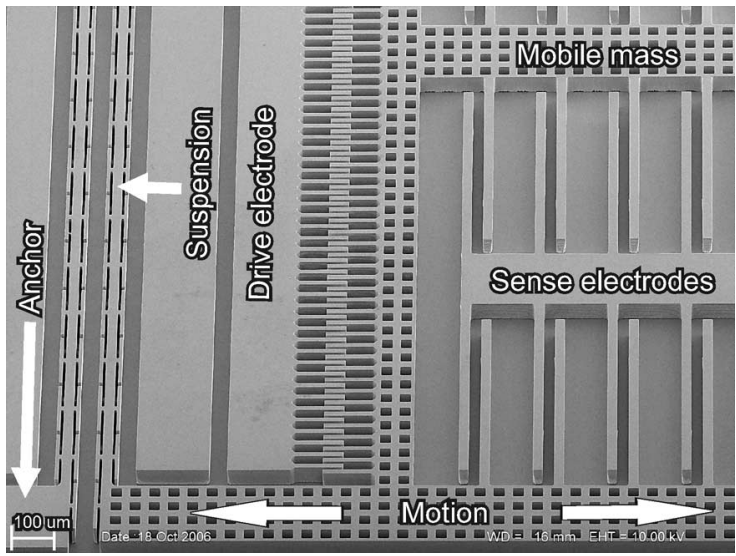

Fig. 4. SEM micrograph of a quarter of a fabricated device.

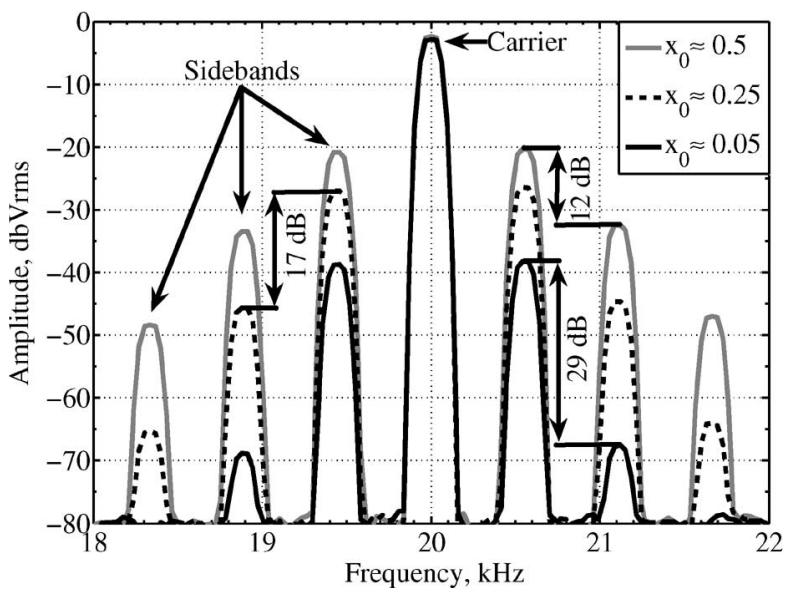

Fig. 5. Experimental spectral measurement of the parallel-plate EAM pick-up signal at different motional amplitudes (see Table I).

50- $\mu$ m-thick device layer were used. AZ-4620 photoresist was spin-coated onto the wafers and patterned using a chromeon-glass mask and a Karl Suss MA-6 exposure system. After photoresist development, the wafers were subjected to a deep reactive-ion etching using a Surface Technology Systems (STS) tool and then released in a HF-acid bath. The minimal gap feature of the process was $5 \mu \mathrm{m}$, and the minimal structural feature was $8 \mu \mathrm{m}$. The nominal gap in the parallel-plate sense capacitor was $25 \mu \mathrm{m}$. It should be noted that the relatively large $25-\mu \mathrm{m}$ gaps were chosen for easier optical tracking of motion during the experiments and are not, otherwise, fundamental to the demonstrated phenomena. A SEM image of a fabricated test resonator is shown in Fig. 4. The resonator had a resonant frequency of $\approx 555 \mathrm{~Hz}$ and a quality factor of $Q \approx 15$ in air.

During the experiment, the test device was driven into linear vibrations using a lateral-comb drive capacitor with a combination of $30-\mathrm{V}$ dc bias with a $0.1-3.5 \mathrm{Vrms}$ ac at $555 \mathrm{~Hz}$. An ac carrier voltage of $5 \mathrm{Vrms}$ at $20 \mathrm{kHz}$ was applied to the mobile mass. A parallel-plate sense capacitor was connected to a transimpedance amplifier, as shown in Fig. 1. The voltage output of the amplifier was fed into a dynamic signal analyzer for data capturing and spectral measurements.

Fig. 5 shows the frequency spectrum of the produced pickup signal at three different amplitudes of motion, which were
TABLE I

SBR Detection of the Normalized Amplitude of Motion $x_{0}(r)$

\begin{tabular}{|c|c|c|}
\hline visually detected $x_{0}$ & measured SBR & $x_{0}$ from Equation (13) \\
\hline$\approx 0.05$ & $-29.2 \mathrm{~dB}=0.347$ & 0.07 \\
\hline$\approx 0.25$ & $-17.5 \mathrm{~dB}=0.133$ & 0.26 \\
\hline$\approx 0.5$ & $-12 \mathrm{~dB}=0.251$ & 0.47 \\
\hline
\end{tabular}

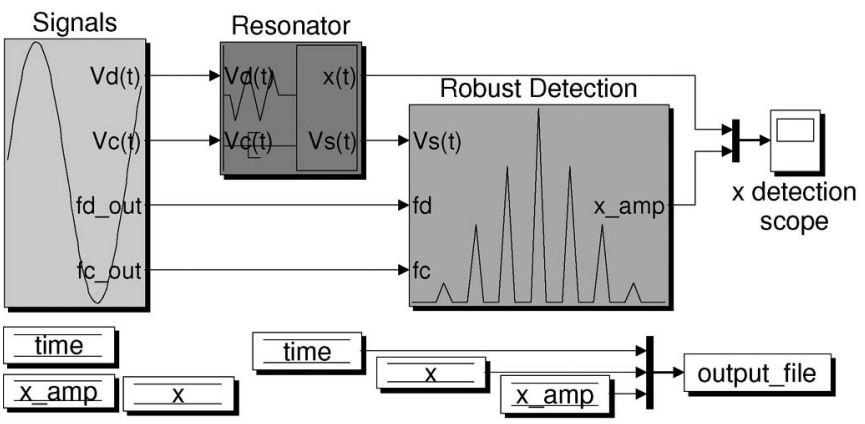

(a)

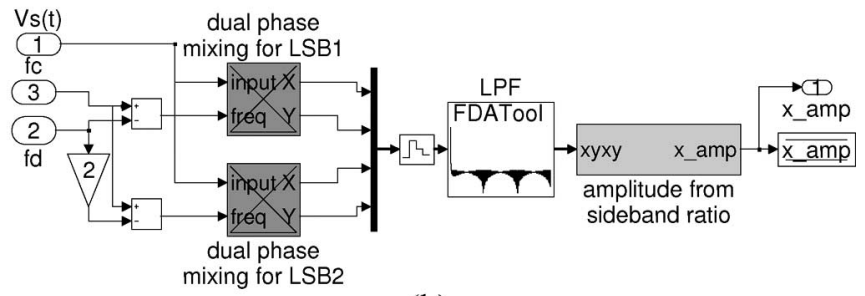

(b)

Fig. 6. Simulink model of a resonator with parallel-plate SBR detection. (a) Top-level schematic. (b) "Robust Detection" block.

visually estimated using a calibrated optical microscope. As expected from (7), multiple sidebands are present in the spectrum, and their amplitudes form a geometric progression (which is seen as a linear decay of amplitudes on a logarithmic scale), while the carrier feed-through stays essentially the same. Table I analyzes the experimental measurements using (13). As anticipated from the theoretical analysis, the amplitudes of motion can be obtained from the ratio of subsequent sidebands without relying on values of any other system parameters.

\section{ReAL-Time Algorithm IMPlementation}

A real-time measurement algorithm based on (12) and (13) was developed and simulated using Simulink software. Fig. 6(a) shows the overall view of the Simulink model. It consists of the following high-level blocks.

1) The "Signals" block generates drive and carrier voltages.

2) The "Resonator" block represents a capacitive resonator. The outputs of this block are the displacement $x(t)$ and the parallel-plate EAM pick-up voltage $V_{\mathrm{s}}(t)$.

3) The "Robust Detection" block extracts the amplitude of motion from the ratio of parallel-plate EAM sidebands.

Fig. 6(b) shows the demodulation procedure, which consists of two steps: amplitude demodulation of two successive sidebands and calculation of the motional amplitude from the ratio of sidebands amplitudes. The "Robust Detection" block includes dual-phase mixing at frequencies of the first and 


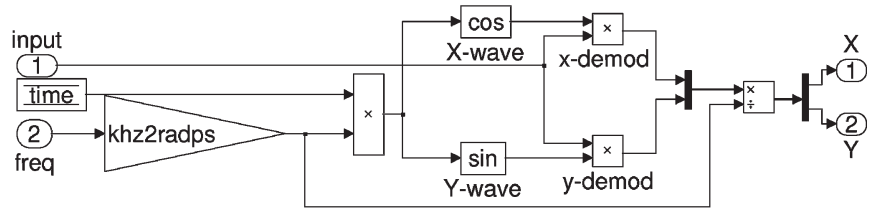

(a)

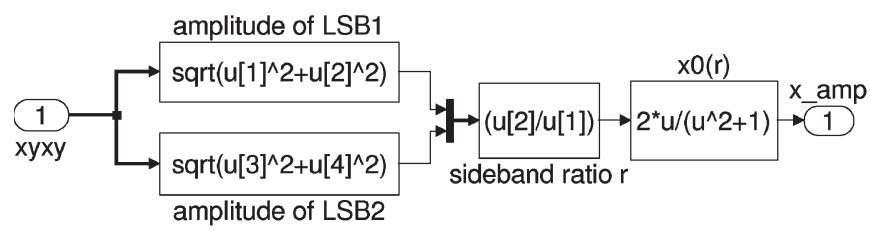

(b)

Fig. 7. Simulink model of a resonator with parallel-plate SBR detection. (a) "Dual-phase mixing" block. (b) "Amplitude from SBR" block.
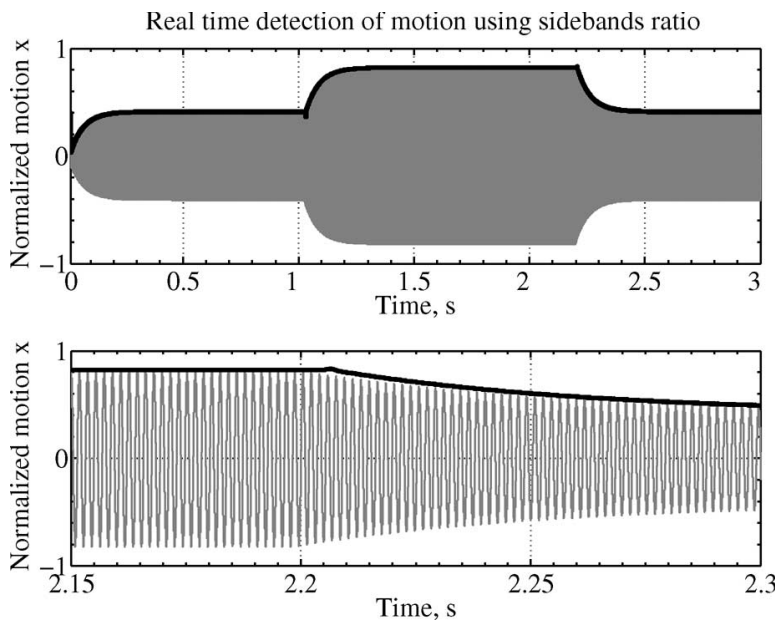

Fig. 8. Parallel-plate SBR detection of motional amplitude, simulation results. The bottom figure is a zoom-in of the top plot. The gray sinusoidal line is the actual motion, and the black envelope line is the result of the demodulation algorithm.

second left sidebands [Fig. 7(a)]. Extraction of the amplitude of motion according to (13) is done by the "amplitude from SBR" block [Fig. 7(b)]. The rest of the model blocks are shown in Appendix II.

The described Simulink model was used to verify feasibility of the proposed real-time SBR-detection method. Fig. 8 shows the results of simulation for a resonator with a quality factor $Q=100$ and a natural frequency of $555 \mathrm{~Hz}$. The carrier voltage was $2 V_{\mathrm{pk}}$ at $10 \mathrm{kHz}$. Other simulation parameters include the nominal sense capacitance $C_{\mathrm{sn}}=1 \mathrm{pF}$ and transimpedance amplification gain of $0.5 \mathrm{M} \Omega$. During the simulation, the driving ac voltage amplitude was switched between 1 and $2 V_{\mathrm{pk}}$, causing changes of the motional amplitude. The results of the simulation confirm feasibility of the SBR-detection algorithm.

\section{DISCUSSION}

\section{A. Differential EAM}

In the case of differential EAM, all even-order sidebands cancel out from the total pick-up signal, while the odd-order sidebands double in amplitude. Similarly, to the regular case, the amplitude of motion $x_{0}$ is calculated using (13); parameter $r$ can be calculated from the ratio of third and first sidebands according to

$$
\begin{aligned}
r\left(x_{0}\right) & =\sqrt{\frac{\left\|V_{\omega_{-(k+2)}}\right\|+\left\|V_{\omega_{(k+2)}}\right\|}{\left\|V_{\omega_{-k}}\right\|+\left\|V_{\omega_{k}}\right\|}} \\
& =\sqrt{\frac{\left\|p_{k+2}\left(x_{0}\right)\right\|}{\left\|p_{k}\left(x_{0}\right)\right\|}} \approx \sqrt{\frac{\left\|V_{\omega_{ \pm(k+2)}}\right\|}{\left\|V_{\omega_{ \pm k}}\right\|}} .
\end{aligned}
$$

\section{B. Detection With Pure DC Voltage}

In some cases, the effect of parasitics is negligible, and capacitive detection is done with a purely dc-biased sense capacitor [12]. In addition, pure dc detection is often used for frequency-response characterization of vibratory devices; in this case, responses of the device under test are collected for different frequencies of the ac driving voltage, and contribution of the parasitics is eliminated using postprocessing [8]. The pure dc detection does not involve any carrier and is described by (7) with $v_{\mathrm{c}}=0$. In this case, the total output voltage is given by

$$
\begin{aligned}
& V(t)=R C_{\mathrm{sn}} V_{\mathrm{dc}} \omega_{\mathrm{d}} \sum_{k=1}^{\infty} 2 k p_{2 k}\left(x_{0}\right) \sin \left(2 k \omega_{\mathrm{d}} t\right) \\
& -R C_{\mathrm{sn}} V_{\mathrm{dc}} \omega_{\mathrm{d}} \sum_{k=1}^{\infty}(2 k+1) p_{2 k+1}\left(x_{0}\right) \cos \left((2 k+1) \omega_{\mathrm{d}} t\right) .
\end{aligned}
$$

The information about the amplitude of motion is carried by the harmonics of the drive frequency. In the conventional linear approach, only the first harmonic is considered and is assumed to be proportional to the amplitude of motion. In the complete nonlinear case, the amplitude $x_{0}$ can be calculated based on the ratio of the subsequent harmonics [(13)]. In turn, the ratio $r$ is easily obtained from the pick-up voltage

$$
r\left(x_{0}\right)=\frac{\left\|p_{k+1}\left(x_{0}\right)\right\|}{\left\|p_{k}\left(x_{0}\right)\right\|}=\frac{k\left\|V_{(k+1) \omega_{\mathrm{d}}}\right\|}{(k+1)\left\|V_{k \omega_{\mathrm{d}}}\right\|}=\frac{\left\|V_{2 \omega_{\mathrm{d}}}\right\|}{2\left\|V_{\omega_{\mathrm{d}}}\right\|} .
$$

In the case of differential detection with dc-biased parallelplate sense capacitors, only the odd-order drive-frequency harmonics are present; the ratio $r$ can be calculated from the total pick-up voltage as

$$
r\left(x_{0}\right)=\sqrt{\frac{k\left\|V_{(k+2) \omega_{d}}\right\|}{(k+2)\left\|V_{k \omega_{\mathrm{d}}}\right\|}}=\sqrt{\frac{\left\|V_{3 \omega_{\mathrm{d}}}\right\|}{3\left\|V_{\omega_{\mathrm{d}}}\right\|}} .
$$

For instance, the ratio of first and third drive-frequency harmonics can be used.

\section{Demodulation}

Both the conventional and the proposed detection methods utilize the amplitudes of certain frequency components of the total pick-up signal. For instance, in the case of carrier modulation, the proposed method uses amplitudes of the two pairs of EAM sidebands, $\left\|V_{\omega_{ \pm 1}}\right\|$ and $\left\|V_{\omega_{ \pm 2}}\right\|$. The detection of these amplitudes is done by mixing with a reference sinusoidal signal followed by a low-pass filter stage to obtain a dc measurement. 
The frequency of the reference waveform should match the frequency of interest. During the operation (either open- or closed-loop), the reference signals for the drive and carrier frequencies $\omega_{\mathrm{d}}$ and $\omega_{\mathrm{c}}$ are available to the control system. Since mixing of reference signals results in the summation of their frequencies, the reference signals for $\omega_{k}=\omega_{\mathrm{c}} \pm k \omega_{\mathrm{d}}$ frequencies of the sidebands can be obtained by mixing $\omega_{\mathrm{d}}$ reference with $\omega_{\mathrm{c}}$ reference once (for the sideband order $k=1$ ) or twice (for $k=2$ ).

\section{Noise Analysis}

In this section, we discuss the practical tradeoffs of random noise performance and accuracy for the proposed measurement method with comparison to the conventional approach. Let us assume that, in addition to the useful frequency components, the total pick-up voltage also contains an additive-white-noise background. This noise level of the signal line propagates through a measurement algorithm resulting in the uncertainty of the produced amplitude measurement.

We denote the effective uncertainty of the sideband amplitude demodulation by $\sigma=\left(C_{\mathrm{sn}} v_{\mathrm{c}} \omega_{\mathrm{c}} R\right) e$, where $e$ is the normalized dimensionless uncertainty. In the conventional linear approach, the amplitude of motion is extracted from the measured amplitudes of the first-order sidebands. In this case, the signal-to-noise ratio (SNR) can be defined as

$$
\mathrm{SNR}=\frac{\left\|V_{\omega_{1}}\right\|+\left\|V_{\omega_{-1}}\right\|}{\sigma}=\frac{\left\|p_{1}\left(x_{0}\right)\right\|}{e} \approx \frac{x_{0}}{e}
$$

and is proportional to the amplitude of motion $x_{0}$ and inversely proportional to the sideband demodulation uncertainty $e$.

For the SBR method, the sidebands demodulation uncertainty $e$ is present in the measured amplitudes of both the firstand second-order sidebands and propagates to the motional amplitude detection uncertainty through (11) and (13). According to these equations, the SBR-based measurement of $x_{0}$, in the presence of demodulation uncertainty, can be expressed as a random variable

$$
\widehat{x}_{0}=\widehat{x}_{0}\left(x_{0}, \widehat{e}_{1}, \widehat{e}_{2}\right)=\frac{2\left(\frac{p_{2}\left(x_{0}\right)+\widehat{e}_{2}}{p_{1}\left(x_{0}\right)+\widehat{e}_{1}}\right)}{\left(\frac{p_{2}\left(x_{0}\right)+\widehat{e}_{2}}{p_{1}\left(x_{0}\right)+\widehat{e}_{1}}\right)^{2}+1}
$$

where $\widehat{e}_{1,2}$ are two uncorrelated random variables with zero mean and equal standard deviation $e$. According to the multivariate case of the law of propagation of uncertainty [13], the uncertainty of the amplitude measurement using the SBR method is defined by

$$
\begin{aligned}
\Delta\left(\widehat{x}_{0}\right) & =e \sqrt{\left.\sum_{i=1}^{i=2}\left(\frac{\partial \widehat{x}_{0}\left(x_{0}, \widehat{e}_{1}, \widehat{e}_{2}\right)}{\partial \widehat{e}_{i}}\right)^{2}\right|_{\widehat{e}_{1,2}=0}} \\
& =\frac{e \sqrt{\left(x_{0}^{2}-1-\sqrt{1-x_{0}^{2}}\right)^{2}\left(1-x_{0}^{2}\right)\left(1+\sqrt{1-x_{0}^{2}}\right)}}{\sqrt{2} x_{0}} .
\end{aligned}
$$

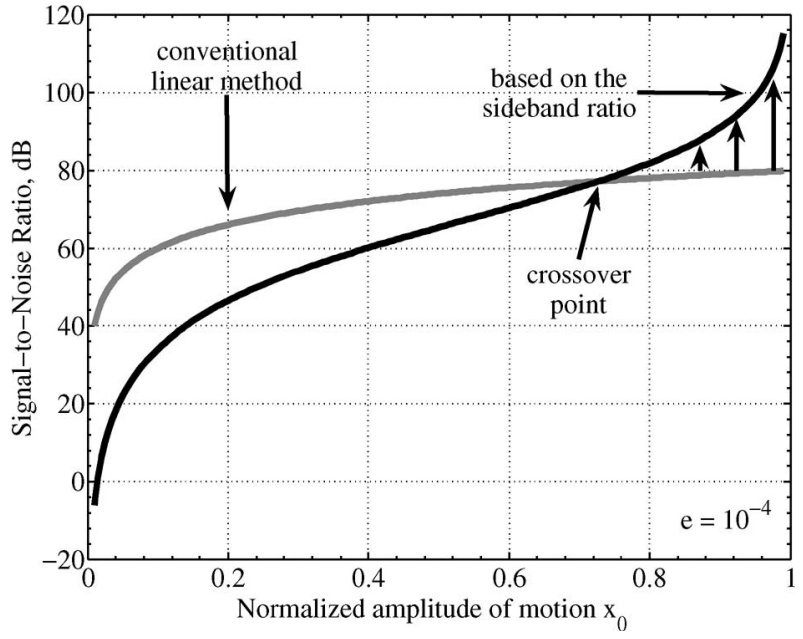

Fig. 9. SNR of the conventional and SBR motional amplitude detection methods for $e=-80 \mathrm{~dB}$.

The SNR of the SBR-detection method can be obtained as

$$
\begin{aligned}
\mathrm{SNR}_{\mathrm{sbr}} & =\frac{x_{0}}{\Delta\left(\widehat{x}_{0}\right)} \\
& =\frac{\sqrt{2} x_{0}^{2}}{e \sqrt{\left(x_{0}^{2}-1-\sqrt{1-x_{0}^{2}}\right)^{2}\left(1-x_{0}^{2}\right)\left(1+\sqrt{1-x_{0}^{2}}\right)}} .
\end{aligned}
$$

The $\mathrm{SNR}_{\mathrm{sbr}}$ is inversely proportional to the initial demodulation uncertainty $e$ and depends on the amplitude of motion $x_{0}$ in a nonlinear fashion. Fig. 9 shows $\mathrm{SNR}_{\text {sbr }}$ with comparison to the conventional linear measurement for $e=10^{-4}=-80 \mathrm{~dB}$ and assuming equal constants $C_{\mathrm{sn}} v_{\mathrm{c}} \omega_{\mathrm{c}} R$. At small amplitudes of motion, SNR of the conventional method is superior due to the very small amplitudes of the secondary sidebands $\left\|V_{\omega_{ \pm 2}}\right\|$ being masked by the noise. However, $\mathrm{SNR}_{\mathrm{sbr}}$ improves much faster with increased amplitude of motion and surpasses the conventional method at a certain crossover point.

In order to facilitate the comparative noise analysis further, we introduce the relative noise figure as

$$
\begin{aligned}
\mathrm{NF}_{\mathrm{sbr}} & =\frac{\mathrm{SNR}}{\mathrm{SNR}_{\mathrm{sbr}}}=\frac{x_{0}}{e} / \frac{x_{0}}{\Delta\left(\widehat{x}_{0}\right)}=\frac{\Delta\left(\widehat{x}_{0}\right)}{e} \\
& =\frac{\sqrt{\left(x_{0}^{2}-1-\sqrt{1-x_{0}^{2}}\right)^{2}\left(1-x_{0}^{2}\right)\left(1+\sqrt{1-x_{0}^{2}}\right)}}{\sqrt{2} x_{0}} .
\end{aligned}
$$

It describes the ratio of uncertainties for the proposed and the conventional methods and is, in fact, independent of the sidebands demodulation uncertainty $e$. As shown in Fig. 10, the relative noise figure is a monotonically decreasing function of $x_{0}$ and crosses unity at a crossover point of $x_{0}=0.72$; for large amplitudes of motion, the proposed method provides up to $30 \mathrm{~dB}$ improvement of the random noise performance. 


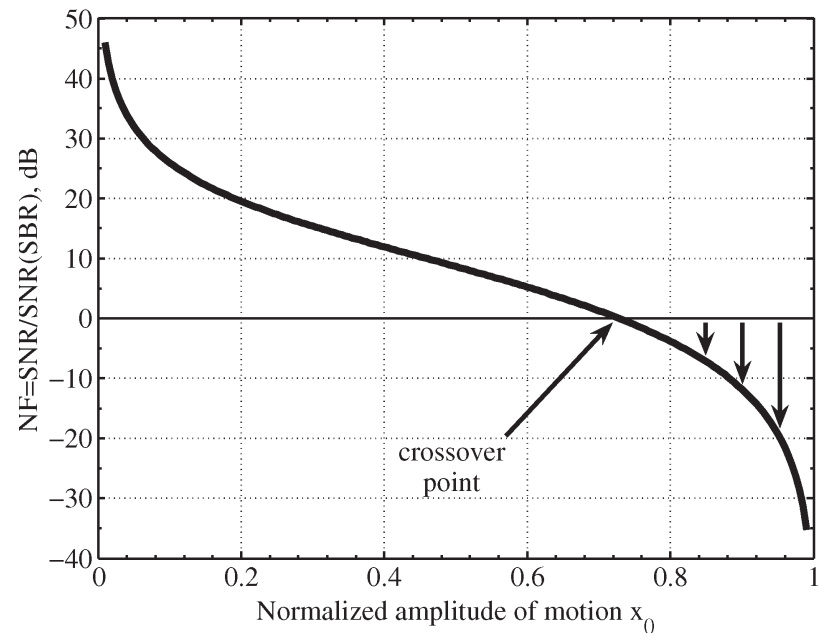

Fig. 10. Noise figure for the SBR-based measurement as compared to the conventional linear method (does not depend on $e$ ).

The presented performance analysis of the SBR method, as compared to the conventional linear approach, is somewhat conservative for the following reasons. Conventional linear detection can be implemented in two alternative ways. First, a linearized model of the parallel-plate capacitors can be used. At large amplitudes of motion, this results in a significant systematic error, which is much more detrimental to the measurement than the random noise. The SBR-based method eliminates this systematic error by using the complete nonlinear model. Second, lateral-comb capacitors with linear capacitance can be used for linear detection. However, the nominal capacitive gradient of combs is typically very small as compared to parallel plates of the same device area. Thus, for the same signal uncertainty $\sigma$, a parallel-plate capacitor has smaller normalized noise $e$, resulting in a smaller amplitude measurement uncertainty and, thus, a more precise measurement.

\section{CONCLUSION}

We presented a novel method of motion detection in capacitive resonant devices. The method constructively utilizes the nonlinearity of parallel-plate capacitors in order to produce an accurate self-calibrated measurement of the motional amplitude in real time. Feasibility of the proposed approach is supported by experimental results and simulations.

In the case of parallel-plate sense capacitor, the pick-up signal contains multiple nonlinear harmonics corresponding to the motional frequency. All these harmonics carry information about the amplitude of the sinusoidal motion and can be used simultaneously to detect the amplitude of motion. Unlike conventional approaches, the proposed SBR-detection procedure does not depend on values of such system parameters as the nominal capacitance, the modulating carrier voltage and frequency, and the gain of the current amplifier. The method also eliminates the systematic error caused by the parallel-plate capacitor nonlinearity and provides up to $30 \mathrm{~dB}$ improvement of the SNR for large amplitudes of motion.

The SBR-detection method is applicable to a variety of dynamic MEMS devices where the detection of the amplitude of sinusoidal vibrations is necessary. The method can be employed with both single-sided and differential schemes using either dc or ac sensing voltages. Depending on the operational range of the motional amplitude, the method can be used in two distinct ways. For devices with large nominal amplitudes of vibration (as compared to the fabrication process resolution of capacitive gap features), the method allows to take advantage of highly sensitive parallel-plate sense capacitors to produce accurate and precise real-time measurements immune to the initial values and fluctuations of the capacitance, probing voltages, and amplification gains. Gyroscopes with lateral-comb actuation and parallel-plate detection of the drive-mode motion, such as [14], are a natural example of such application.

For microstructures with intrinsically small deflections, such as sense-mode resonators of vibratory gyroscopes, the method can be used for automatic identification and self-calibration. In this case, a dedicated procedure can be periodically executed (for instance, during the start-up) by the device electronics, during which the vibration is actuated and detected using the conventional and the SBR method simultaneously. The detection bandwidth during this procedure can be significantly reduced to suppress the SBR method random noise present at small amplitudes of motion. By relating the outputs of both detection methods, the deflection-to-voltage scaling factors of the device can be automatically identified and used throughout the realtime operation. Utilization of the SBR-detection method allows for easier recalibration of small-amplitude dynamic MEMS by eliminating any external equipment from the automatic identification and calibration procedure.

\section{APPENDIX I \\ DERIVATIONS OF FOURIER SERIES FOR PARALlEL-Plate CAPACITANCE}

Parallel-plate variable sense capacitance is $C_{\mathrm{s}}(t)=C_{\mathrm{sn}} /$ $\left(1-x_{0} \sin \left(\omega_{\mathrm{d}} t\right)\right)$. Since, physically, $x_{0}<1$, Taylor expansion method can be used to obtain a precise infinite-series expansion for the nonlinear parallel-plate capacitance

$$
C_{\mathrm{s}}(t)=C_{\mathrm{sn}} \sum_{n=0}^{\infty}\left(x_{0} \sin \left(\omega_{\mathrm{d}} t\right)\right)^{n} .
$$

We use Euler's formula to convert from powers of sinusoids to harmonics of multiple frequencies

$$
\sin \left(\omega_{\mathrm{d}} t\right)=\frac{1}{2 i}\left(e^{i \omega_{\mathrm{d}} t}-e^{-i \omega_{\mathrm{d}} t}\right)
$$

and Newton's binomial theorem to expand these terms

$$
(a+b)^{n}=\sum_{k=0}^{n} C(n, k) a^{n-k} b^{n}
$$

where $C(n, k)=C_{k}^{n}=n ! / k !(n-k) !$ are Newton's binomial coefficients. We substitute the harmonic terms in (25) with complex exponents using (26). Then, each term in the sum can be expanded according to (27). Terms of odd and even orders are then collected in two distinct groups. Finally, (26) can be used again (this time, in reverse direction) to convert terms 
back from complex exponents to the regular sine and cosine harmonics. The sense capacitance (normalized with respect to the nominal sense capacitance) is obtained as

$$
\begin{aligned}
& \frac{C_{\mathrm{s}}(t)}{C_{\mathrm{sn}}} \\
& =\sum_{n=0}^{\infty} x_{0}{ }^{2 n} \frac{(-1)^{n}}{2^{2 n-1}} \sum_{k=0}^{n}(-1)^{n-k} C_{n-k}^{2 n} \cos \left(2 k \omega_{\mathrm{d}} t\right) \\
& \quad+\sum_{n=0}^{\infty} x_{0}{ }^{2 n+1} \frac{(-1)^{n}}{2^{2 n}} \\
& \quad \times \sum_{k=0}^{n}(-1)^{n-k} C_{n-k}^{2 n+1} \sin \left((2 k+1) \omega_{\mathrm{d}} t\right) .
\end{aligned}
$$

Equation (28) gives a power series expansion of sense capacitance $C_{\mathrm{s}}(t)$ with respect to the normalized motional amplitude $x_{0}$. The coefficients in front of each of the $x_{0}$ power terms are formed by finite sum of drive frequency harmonics $\left(n \omega_{\mathrm{d}}\right)$ with binomial coefficient weights. We swap the summation order by algebraically regrouping terms in (28)

$$
\begin{aligned}
\frac{C_{\mathrm{s}}(t)}{C_{\mathrm{sn}}}= & \sum_{k=0}^{\infty} \cos \left(2 k \omega_{\mathrm{d}} t\right) \\
& \times \sum_{n=k}^{\infty}\left\{\frac{(-1)^{2 n-k}}{2^{2 n-1}} C_{n-k}^{2 n} x_{0}^{2 n}\right\} \\
& +\sum_{k=0}^{\infty} \sin \left((2 k+1) \omega_{\mathrm{d}} t\right) \\
& \times \sum_{n=k}^{\infty}\left\{\frac{(-1)^{2 n-k}}{2^{2 n}} C_{n-k}^{2 n+1} x_{0}^{2 n+1}\right\} .
\end{aligned}
$$

This equation can be rewritten as a Fourier series for the variable sense capacitance

$$
\begin{aligned}
C_{\mathrm{s}}(t)=C_{\mathrm{sn}} \sum_{k=0}^{\infty} \cos \left(2 k \omega_{\mathrm{d}} t\right) p_{2 k}\left(x_{0}\right) \\
+C_{\mathrm{sn}} \sum_{k=0}^{\infty} \sin \left((2 k+1) \omega_{\mathrm{d}} t\right) p_{2 k+1}\left(x_{0}\right)
\end{aligned}
$$

where the $p_{k}\left(x_{0}\right)$ Fourier coefficients in front of the harmonic terms are given by the following infinite power series:

$$
\begin{aligned}
p_{0}\left(x_{0}\right) & =\sum_{n=0}^{\infty} \frac{C_{n}^{2 n}}{2^{2 n}} x_{0}^{2 n}, \quad \text { and for } k=0,1,2, \ldots, \infty \\
p_{2 k+1}\left(x_{0}\right) & =(-1)^{k} \sum_{n=k}^{\infty} \frac{C_{n-k}^{2 n+1}}{2^{2 n}} x_{0}^{2 n+1} \\
p_{2 k}\left(x_{0}\right) & =(-1)^{k} \sum_{n=k}^{\infty} \frac{C_{n-k}^{2 n}}{2^{2 n-1}} x_{0}^{2 n} .
\end{aligned}
$$

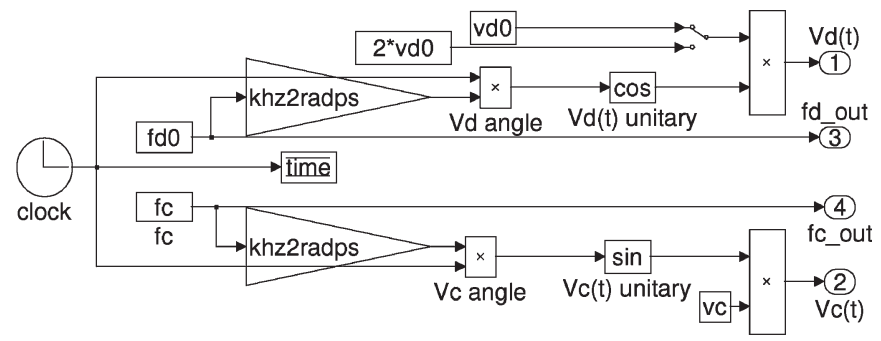

(a)

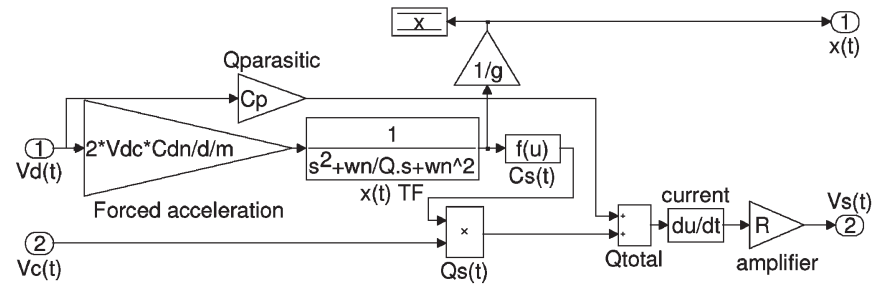

(b)

Fig. 11. Simulink model of a resonator with parallel-plate SBR detection. (a) "Signals" block. (b) "Resonator" block.

Closed-form expressions for $p_{k}\left(x_{0}\right)$ are found as

$$
\begin{aligned}
\left\|p_{k}\left(x_{0}\right)\right\| & =\frac{2}{\sqrt{1-x_{0}^{2}}}\left(\frac{x_{0}}{\left(1+\sqrt{1-x_{0}^{2}}\right)}\right)^{k} \\
& =2 p_{0}\left(x_{0}\right) \quad\left(\frac{x_{0}}{\left(1+\sqrt{1-x_{0}^{2}}\right)}\right)^{k} .
\end{aligned}
$$

\section{APPENDIX II}

\section{Additional Blocks of the Simulink Model}

Fig. 11(a) and (b) show content of the "Signals" and the "Resonator" blocks of the Simulink model, discussed in Section VI. The "Signals" block generates drive $V_{\mathrm{d}}(t)$ and carrier $V_{\mathrm{c}}(t)$ voltages and a common time reference for the system. In the "Resonator" block, input drive voltage is converted into electrostatic force, which excites the motion. Displacement $x(t)$ is used to calculate the total charge of the parallel-plate sense capacitor. The total output current is calculated as a sum of parasitic and sense currents. The block outputs the total amplified voltage.

\section{ACKNOWLEDGMENT}

The experiments presented in this paper were conducted at the UCI MicroSystems Laboratory. The authors would like to thank the UCI Integrated Nanosystems Research Facility (INRF) for the help with the fabrication of the test devices and A. R. Schofield of the UCI MicroSystems Laboratory for the insightful discussions.

\section{REFERENCES}

[1] N. Yazdi, F. Ayazi, and K. Najafi, "Micromachined inertial sensors," Proc. IEEE, vol. 86, no. 8, pp. 1640-1659, Aug. 1998.

[2] L. Lin, R. T. Howe, and A. P. Pisano, "Microelectromechanical filters for signal processing," J. Microelectromech. Syst., vol. 7, no. 3, pp. 286-294, Sep. 1998. 
[3] C. T.-C. Nguyen, "Frequency-selective MEMS for miniaturized lowpower communication devices," IEEE Trans. Microw. Theory Tech., vol. 47, no. 8, pp. 1486-1503, Aug. 1999.

[4] A.-C. Wong and C. T.-C. Nguyen, "Micromechanical mixer-filters ("mixlers")," J. Microelectromech. Syst., vol. 13, no. 1, pp. 100-112, Feb. 2004.

[5] G. Stemme, "Resonant silicon sensors," J. Micromech. Microeng., vol. 1, no. 2, pp. 113-125, Jun. 1991.

[6] J. Cao and C.-C. Nguyen, "Drive amplitude dependence of micromechanical resonator series motional resistance," in Dig. Tech. Papers, 10th Int. Conf. Solid-State Sens., Actuators, Sendai, Japan, Jun. 7-10, 1999, pp. 1826-1829.

[7] C. Acar and A. M. Shkel, "Structurally decoupled micromachined gyroscopes with post-release capacitance enhancement," J. Micromech. Microeng., vol. 15, no. 5, pp. 1092-1101, May 2005.

[8] A. Trusov, C. Acar, and A. M. Shkel, "Comparative analysis of distributed mass micromachined gyroscopes fabricated in SCS-SOI and EFAB," in Proc. SPIE Smart Struct. Mater.: Sens. Smart Struct. Technol. Civil, Mech., Aerosp. Syst., San Diego, CA, Feb. 26-Mar. 2, 2006, pp. 61742A.1-61742A.12.

[9] B. Cagdaser, A. Jog, M. Last, B. S. Leibowitz, L. Zhou, E. Shelton, K. S. Pister, and B. E. Bposer, "Capacitive sense feedback control for MEMS beam steering mirrors," in Proc. Solid-State Sens., Actuators, Microsyst. Workshop, Hilton Head, SC, Jun. 6-10, 2004, pp. 348-351.

[10] A. A. Trusov and A. M. Shkel, "Capacitive detection in resonant MEMS with arbitrary amplitude of motion," J. Micromech. Microeng., vol. 17, no. 8, pp. 1583-1592, Aug. 2007.

[11] P. Horowitz and W. Hill, The Art of Electronics. Cambridge, U.K.: Cambridge Univ. Press, 1989

[12] J. A. Geen, S. J. Sherman, J. F. Chang, and S. R. Lewis, "Single-chip surface-micromachined integrated gyroscope with $50 \mathrm{deg} / \mathrm{hour}$ root Allan variance," in Proc. IEEE Int. Solid-State Circuits Conf., San Francisco, CA, Feb. 3-7, 2002, pp. 346-539.

[13] B. N. Taylor and C. E. Kuyatt, "Guidelines for evaluating and expressing the uncertainty of NIST measurement results," Nat. Inst. Standards and Technol., Gaithersburg, MD, Tech. Rep., Note 1297, Sep. 1994.

[14] A. R. Schofield, A. A. Trusov, C. Acar, and A. M. Shkel, "Anti-phase driven rate gyroscope with multi-degree of freedom sense mode," in Proc. Int. Conf. Solid-State Sens., Actuators, Microsyst., TRANSDUCERS, Lyon, France, Jun. 10-14, 2007, pp. 1199-1202.

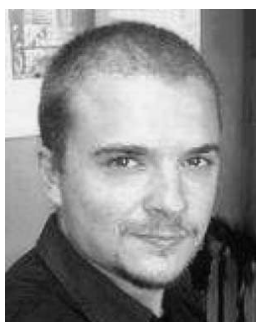

Alexander A. Trusov (S'06) received the Diploma degree in applied mathematics and mechanics from Moscow State University, Moscow, Russia, in 2004, and the M.S. degree in mechanical and aerospace engineering from the University of California, Irvine, in 2006, where he is currently working toward the Ph.D. degree.

His research interests include the design, fabrication, and characterization of inertial microelectromechanical systems (MEMS) and the development of novel actuation, detection, signal processing, and control schemes for dynamic MEMS

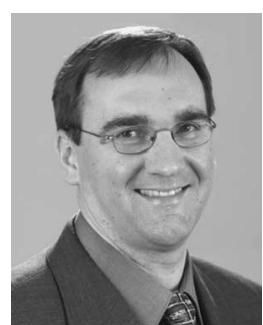

Andrei M. Shkel (S'95-A'98) received the Diploma degree (with excellence) in mechanics and mathematics from Moscow State University, Moscow, Russia, in 1991, and the Ph.D. degree in mechanical engineering from the University of Wisconsin, Madison, in 1997.

Following receipt of the Ph.D. degree, he was with the Berkeley Sensor and Actuator Center as a Postdoctoral Researcher. He then held research and consulting positions in several high-tech and venture companies. Since 2000, he has been with the Faculty of the University of California, Irvine (UCI), where he has been an Associate Professor with the Department of Mechanical and Aerospace Engineering. He is also the Director of the UCI Micro-Systems Laboratory. His professional interests, which are reflected in more than 100 publications, include solid-state sensors and actuators, microelectromechanical-system-based neuroprosthetics, sensor-based intelligence, and control theory. He is the co-inventor of eight U.S. and international patents (with 15 patents pending).

Dr. Shkel is a member of the American Society of Mechanical Engineers, Institute of Navigation, and International Society for Optical Engineers (SPIE). $\mathrm{He}$ is an Editor of the IEEE/ASME Journal of Microelectromechanical Systems and was a Guest Editor for two Special Issues of the IEEE SENSORS Journal, a General Chair of the 2005 IEEE Sensors Conference, and a Vice General Chair and a Publications Chair of the 2002 and 2004 IEEE Sensors Conferences. He was the recipient of the 2006 Research Award of the UCl School of Engineering, a 2005 National Science Foundation CAREER Award, the 2002 George E. Brown, Jr., Award, and a 2001 Fellowship from the Japanese Advanced Science Institute. 